\title{
Netrin-1 Signaling Regulates De Novo Protein Synthesis of $\kappa$ Opioid Receptor by Facilitating Polysomal Partition of Its mRNA
}

\author{
Nien-Pei Tsai, Jing Bi, Horace H. Loh, and Li-Na Wei \\ Department of Pharmacology, University of Minnesota Medical School, Minneapolis, Minnesota 55455
}

The expression of $\kappa$ opioid receptor (KOR) is subjected to both transcriptional and posttranscriptional controls. We report that KOR translation is regulated by netrin-1 in primary neurons of dorsal root ganglion (DRG) and in P19 embryonal carcinoma cells. Without stimulation, a significant portion of KOR mRNA is maintained in a dormant state and partitions in the translationally inactive, postpolysomal fraction. During netrin-1 stimulation, which activates its downstream target focal adhesion kinase (FAK), KOR mRNA rapidly partitions to the translationally active polysomal fraction. Functionally, the newly synthesized KOR proteins in DRG neurons are able to bind to specific ligands. This report describes the first example of netrin-1 signaling in the translational control of a drug receptor KOR, which involves the mediator of netrin-1, FAK, and a novel mechanism that enhances the association of target mRNA with polysomes for translational activation.

Key words: $\kappa$ opioid receptor; netrin-1; FAK; translational regulation; dorsal root ganglia neuron; polysomal partition

\section{Introduction}

Opioid receptors bind to opioid drugs or endogenous opioid ligands to affect pain sensation, consciousness, and autonomic functions. Three opioid receptor types, $\mu, \delta$, and $\kappa$, are known, and they belong to the super family of G-protein-coupled receptors (Minami and Satoh, 1995; Law et al., 1999; Pasternak, 2004). Regulation of their expression has been extensively examined, primarily at the level of gene transcription regulated by vitamin A, cytokines (Bi et al., 2001; Park et al., 2002; Wei and Loh, 2002; $\mathrm{Hu}$ et al., 2004), and specific transcription factors (Kraus et al., 2001; Kim et al., 2004). They also often involve chromatin remodeling events that occur over a period of time and are more permanent (Park et al., 2005). However, when a more rapid response in receptor expression is needed, it could become very inefficient by changing their transcriptional programs. This can be especially a challenge for neurons whose transcription programs often are established and less refractive to stimuli or external factors. Our recent studies uncovered potential regulatory events for the expression of KOR at posttranscriptional levels (Wei et al., 2000; Bi et al., 2003), which could potentially equip neurons with a more rapid and probably transient mechanism to respond to environmental signals.

Posttranscriptional regulation of $\kappa$ opioid receptor (KOR) re-

Received May 2, 2006; revised Aug. 7, 2006; accepted Aug. 10, 2006.

This work is supported in part by National Institutes of Health Grants DA11190, DK54733, DK60521, and K02DA13926 (L.-N.W.) and DA00564, DA01583, DA11806, and K05-DA70554 (H.H.L.). We thank Dr. Kenneth Yamada (National Institute of Dental and Craniofacial Research, Bethesda MD) for providing FAK constructs. We also thank Kathleen L Colten for great help.

Correspondence should be addressed to Li-Na Wei, Department of Pharmacology, University of Minnesota Medical School, 6-120 Jackson Hall, 321 Church Street SE, Minneapolis, MN 55455. E-mail: weixx009@umn.edu. DOI:10.1523/JNEUROSCI.3014-06.2006

Copyright $\odot 2006$ Society for Neuroscience $\quad$ 0270-6474/06/269743-07\$15.00/0 quires its $5^{\prime}$ and $3^{\prime}$ - untranslated regions (UTRs) (Wei et al., 2000; $\mathrm{Hu}$ et al., 2002). However, several questions remain to be answered. What kind of factors could affect KOR protein expression in neurons? What UTR sequences are responsive to environmental factors? What is the mechanism mediating this type of regulation? Recent studies have demonstrated translational regulation in neurons, but direct evidence for translational regulation of nonstructural- or nongrowth-related neuronal proteins is scarce. We found that netrin-1 exerted a rapid and significantly stimulating effect on KOR protein level in KOR-expressing primary neurons and P19 cells. Netrin-1 is an axon guidance cue (Kennedy et al., 1994) that acts on its membrane receptor deleted in colorectal cancer (DCC). DCC interacts with focal adhesion kinase (FAK) (Li et al., 2004; Liu et al., 2004; Ren et al., 2004), resulting in axon attraction and outgrowth. Netrin-1 also stimulates protein synthesis in chemotropic responses through the p42/p44 mitogen-activated protein kinase (MAPK) signaling pathway (Campbell and Holt, 2001, 2003), which is required during resensitization of retinal axons (Piper et al., 2005). However, it is unclear whether and how netrin-1 affects the expression of membrane receptors.

Here we report that netrin-1 rapidly stimulates KOR translation in primary dorsal root ganglion (DRG) neurons and P19 embryonal carcinoma cells by stimulating FAK phosphorylation, which triggers translocation of KOR mRNA from the translationally silent post-polysomal fraction to the translationally active polysomal fraction. Without netrin-1 signals, a significant portion of KOR mRNA is retained in a dormant state in the postpolysomal fraction. During stimulation by netrin-1, KOR mRNA is released to the translationally active polysomal fraction. This report describes the first example of netrin-1 signaling in the translational control of a membrane receptor KOR, which in- 
volves its mediator FAK and uses a novel mechanism that induces differential mRNA partitioning for translational regulation.

\section{Materials and Methods}

Reagents. Monoclonal and polyclonal antibodies were purchased from Santa Cruz Biotechnology (Santa Cruz, CA) (anti-KOR), Abcam (Cambridge, MA) (anti-KOR), Sigma (St. Louis, MO) (anti-Tau), Cell Signaling Technology (Beverly, MA) [anti-eukaryotic initiation factor 4E (eIF4E)], and Upstate Biotechnology (Lake Placid, NY) (anti-FAK, anti- $\alpha$-actin, and anti-phospho-Tyr). Recombinant netrin-1 was from R \& D Systems (Minneapolis, MN). Biotinlabeled dynorphin and HiLyte Fluor 555conjugated streptavidin were from AnaSpec (San Jose, CA). U69,593 [(5 $\alpha, 7 \alpha, 8 \beta)-(+)-N$-methyl$N$-[7-(1-pyrrolidinyl)-1-oxaspiro[4,5] dec-8yl]benzenacetamide] and nor-binaltorphimine (nor-BNI) were from Sigma. ${ }^{3} \mathrm{H}-\mathrm{U} 69,593$ was from PerkinElmer (Wellesley, MA). Gene-specific small interfering RNAs (siRNAs) were from Qiagen (Valencia, CA).

Plasmid constructions, cell cultures, and reporter assay. The interleukin 2 receptor (IL2R), IL2R-FAK, and IL2R-FAK-Y397F were kind gifts from Dr. Kenneth Yamada (National Institute of Dental and Craniofacial Research, Bethesda, MD). FAK silencing constructs were generated in specialized siRNA vector IMG-800 (Imgenex, Sorrento Valley, CA). In brief, synthesized oligonucleotides (FAK, 5' -TCG ACA GGT GAA GAG TGA CTA CAT GGG AAT TCG CAT GTA GTC ACT CTT CAC CTG TTT TT- ${ }^{\prime}$ and $5^{\prime}$-CTA GAA AAA CAG GTG AAG AGT GAC TAC ATG CGA ATT CCC ATG TAG TCA CTC TTC ACC TG-3') were selfannealed and ligated into the IMG- 800 vector.

(A)
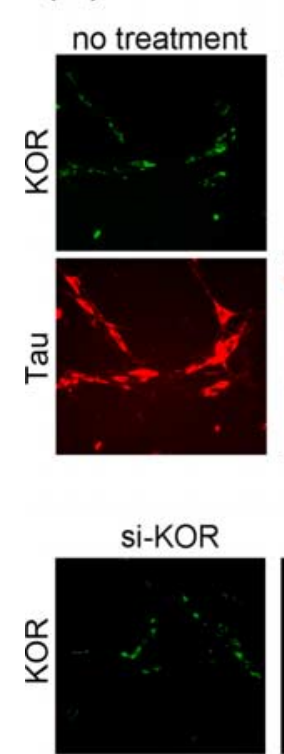

si-KOR plus

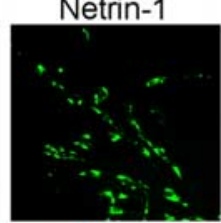

Netrin-1 plus Netrin-1 plus

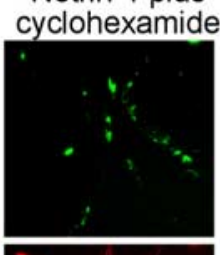

puromycin
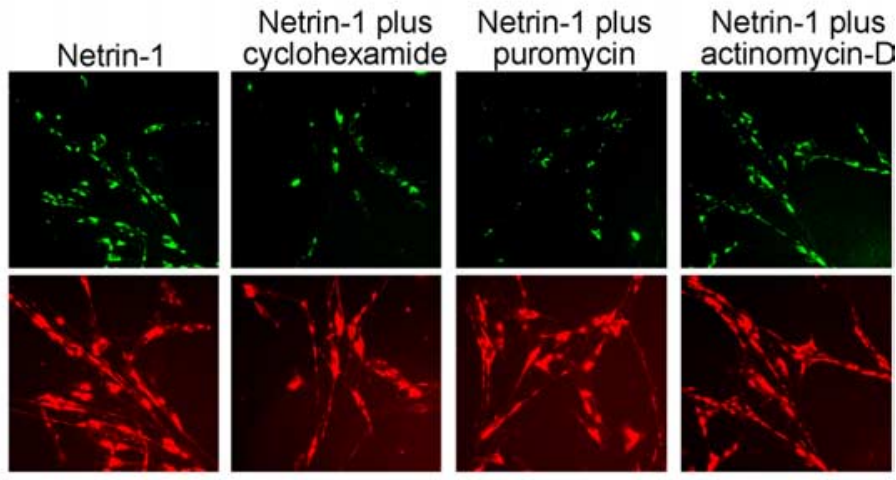

(B)
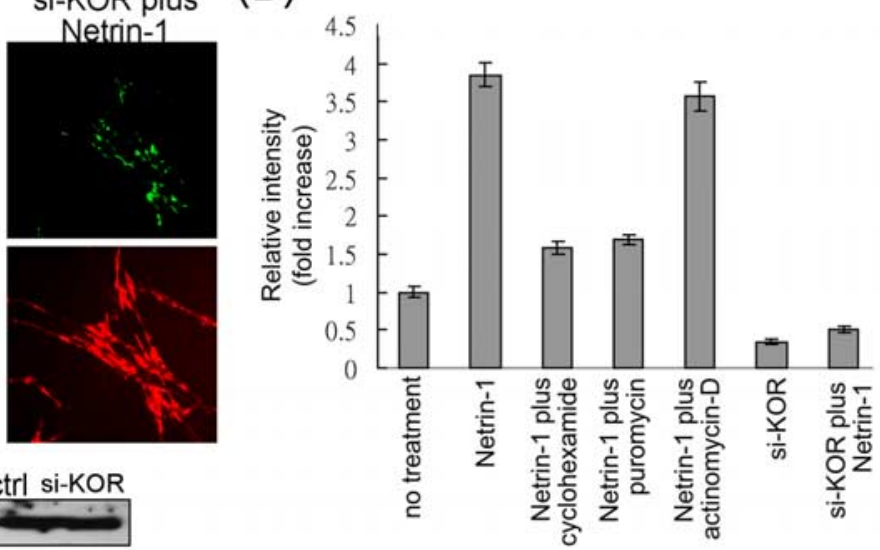

The intact, $5^{\prime}$-UTR or $3^{\prime}$-UTR deleted KOR

translational reporter were constructed with primer pairs specific to KOR 5'-UTR and 3'-UTR sequences (5'-ACT CTC GAG GCG CAC CTT GCT GAT CCC-3', 5' -ACT CCA TGG TGA GCG CTG CAG-3'; and $5^{\prime}$ - CCA TCT AGA CTA GTC GTG GAA ATG TCT TC-3', 5' -ACC GTC GAC CTC CAG CAG TGT CAA GTT GAC-3'), digested with XhoI/ $\mathrm{NcoI}$ or $\mathrm{XbaI} / \mathrm{SalI}$, and ligated into a TK promoter in pGL3-Basic vector (Promega, Madison, WI). DRG neurons were prepared as described previously (Zheng et al., 2001; Stucky et al., 2002; Bi et al., 2003) and plated on $0.1 \mu \mathrm{g} / \mu \mathrm{l}$ poly-D-lysine-coated plates. P19 embryonal carcinoma cells were prepared as described previously (Park et al., 2002). Reporter assay was performed also as described previously (Hu et al., 2002). Three independent experiments were conducted for each assay.

Protein preparation, Western blotting, and immunohistochemistry. Protein samples from P19 cells were prepared as described previously (Park et al., 2002). In brief, cell pellet was resuspended in immunoprecipitation buffer and sonicated. Equal amount of proteins were added into the gel loading buffer and resolved on an $8 \%$ SDS-PAGE. Western blots of various antigens were performed as described previously (Tsai et al., 2006). Immunohistochemistry was conducted as described (Bi et al., 2003) with anti-KOR (1:200) or anti-Tau (1:500) antibodies. To quantify the fluorescent signals, 15 cells were randomly chosen for the measurement of pixel intensity that was obtained with the help of Image ProPlus software (Media Cybernetics, Silver Spring, MD). To exclude uneven branches of cells, the readings were taken only from whole cell bodies. To avoid pixel saturation, the intensity was measured on images of short exposure times. The specific intensities were obtained by normalizing KORspecific signals against Tau-specific signals in the same field. The mean from the control set of cells was given an arbitrary value of 1 for the determination of the relative intensity of each treated culture.

$R N A$ preparation, reverse transcription- $P C R$, and Southern blot analysis. Total RNA was isolated using TRIzol reagent (Invitrogen, Carlsbad, CA) and dissolved in DEPC- $\mathrm{H}_{2} \mathrm{O}$. For reverse transcription (RT) reactions, 2 $\mu \mathrm{g}$ of RNA was used to synthesize cDNA (Qiagen). Specific primer pairs for PCR amplification were as follows: KOR, $5^{\prime}$-CAT CAT CAG GAA ACT GCA- $3^{\prime}$ and $5^{\prime}{ }^{\prime}$ TGG TCA TGT TTG TCA TC- $3^{\prime}$; $\alpha$-actin, $5^{\prime}$-TGG CCT TAG GGT GCA GGG-3' and $5^{\prime}$-GTG GGC CGC TCT AGG CAC CA- $3^{\prime}$ ). Southern blot and semiquantitative analyses of RT-PCR products were performed as described previously (Wei et al., 2000; Bi et al., 2001) and repeated at least three times for each experiment.

Metabolic labeling. P19 cells were cultured in the presence of ${ }^{35} \mathrm{~S}$ labeled Met/Cys (MP Biomedicals, Irvine, CA) for different time intervals. Cells were sonicated and applied to immunoprecipitation. Samples were resolved by $8 \%$ SDS-PAGE and exposed to a PhosphorImager (Molecular Dynamics, Sunnyvale, CA) for quantification.

Ligand binding assay. For in situ ligand binding, primary DRG neurons were first treated with or without $0.2 \mu \mathrm{g} / \mathrm{ml}$ netrin- 1 for $30 \mathrm{~min}$, followed by the addition of $1 \mu \mathrm{m}$ biotin-dynorphin along with or without $2 \mu \mathrm{g} / \mathrm{ml}$ cyclohexamide, $10 \mu \mathrm{g} / \mathrm{ml}$ puromycin, or $2 \mu \mathrm{g} / \mathrm{ml}$ actinomycin-D for another $20 \mathrm{~min}$. After washing, cells were incubated with goat serumdiluted streptavidin, extensively washed, and observed under an inverted microscope (Nikon, Tokyo, Japan). For competition, 50 nM nor-BNI, 10 nм U69,593, or 50 nм unlabeled dynorphin was added along with biotindynorphin. For the quantitative ligand binding assay, primary DRG neurons were suspended in medium and incubated with unlabeled dynor- 
(A)
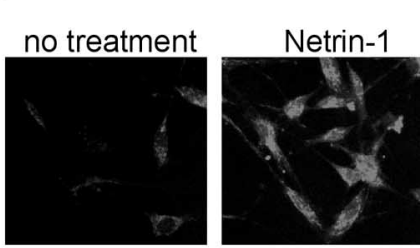

Netrin-1 plus

cyclohexamide

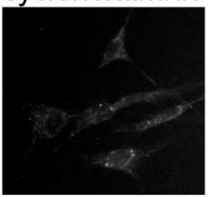

Netrin-1 plus

puromycin

Netrin-1 plus nor-BNI

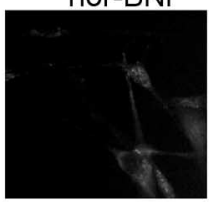

Netrin-1 plus

Netrin-1 plus

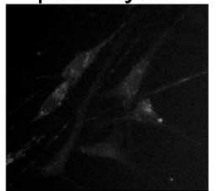

U69,593
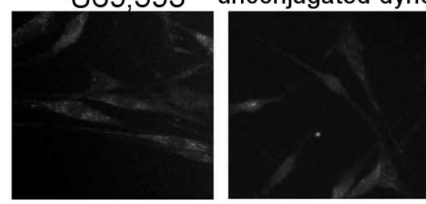

(B)
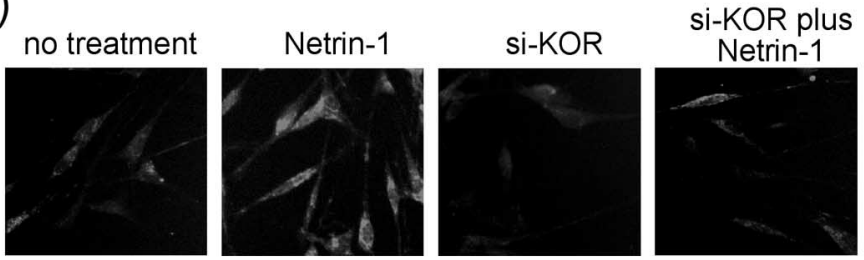

(C)

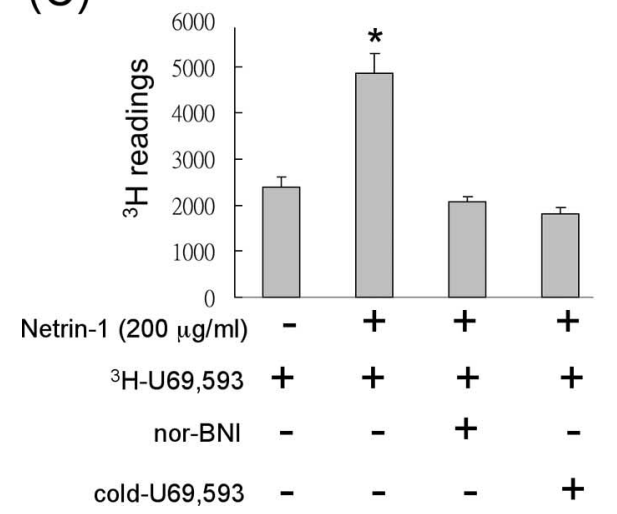

(D)

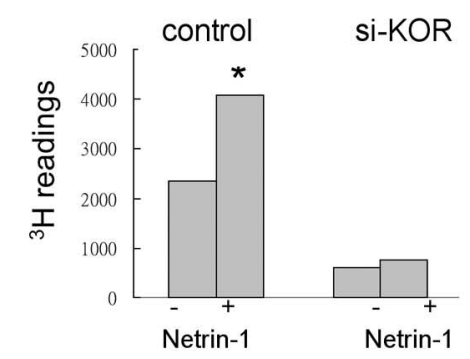

Figure 2. Increased functional KOR in DRG neurons during netrin-1 stimulation. $A$, In situ ligand binding of primary DRG neurons. Biotin-labeled dynorphin was added to control cells, and images of cells treated with netrin-1, netrin-1 plus cyclohexamide, netrin-1 plus puromycin, and netrin-1 plus actinomycin-D are shown in the top row. Images of cells treated with netrin-1 plus nor-BNI, netrin-1 plus U69,593, and netrin-1 plus unconjugated dynorphin are shown in the bottom row. Cells were incubated with HiLyte Fluor 555-conjugated streptavidin and observed under an inverted microscope. $\boldsymbol{B}$, DRG neurons with endogenous KOR knockdown by siRNA were also treated with netrin-1 and detected for specific ligand binding. C, Quantitative ligand binding assays of primary DRG neurons using ${ }^{3} \mathrm{H}$-labeled U69,593 in the presence of various competing KOR ligands $\left({ }^{*} p<0.05\right)$. $D$, Quantitative ligand binding assays of primary DRG neurons with or without endogenous KOR knockdown by siRNA $\left({ }^{*} p<0.05\right)$.

phin for $10 \mathrm{~min}$. Culture medium was changed into a fresh medium containing $0.2 \mu \mathrm{g} / \mathrm{ml}$ netrin-1 for $30 \mathrm{~min} .{ }^{3} \mathrm{H}-\mathrm{U} 69,593$ was then added along with or without nor-BNI or unlabeled U69,593 for another $20 \mathrm{~min}$. Cell pellet was collected and ${ }^{3} \mathrm{H}$ counts were determined. For ligand binding in KOR-silenced DRG neurons, cultures were transfected with KOR siRNA for $48 \mathrm{~h}$ and subjected to binding assays. All binding assays were done at least three times to determine the mean values.

Polysome fractionation and RNA immunoprecipitation. Polysome fractionation was conducted as described previously (Mukhopadhyay et al., 2003). In brief, P19 cell extracts were layered onto the polysomal buffer [10 mм 3-( $N$-morpholino)-propanesulfonic acid, $250 \mathrm{~mm} \mathrm{NaCl}, 2.5 \mathrm{~mm}$ MgOAc, $0.5 \%$ Nonidet P- 40 , and $50 \mu \mathrm{g} / \mathrm{ml}$ cyclohemaxide]. The polysomes were collected by ultracentrifugation at $100,000 \times g$ for $1 \mathrm{~h}$. The supernatant was collected as the post-polysomal fraction. Total RNA was isolated from polysomal and post-polysomal fractions with TRIzol reagent. For RNA immunoprecipitation, cell extracts were subjected to protein immunoprecipitation as described previously (Gupta et al., 2005). After precipitation, TRIzol was applied to the precipitated protein
G beads. Specific mRNAs were then detected by semiquantitative RT-PCR, followed by Southern blot analyses.

\section{Results}

Translation of endogenous KOR is activated by netrin-1 in DRG neurons

KOR mRNA is detected in numerous neurons, including DRG neurons and P19 embryonal carcinoma cells, as well as their differentiated neurons (Bi et al., 2001). In the search for extracellular signals/factors such as growth factors, hormones, and opioids that could possibly affect the expression of KOR protein, we found netrin-1 to be most interesting. As shown in Figure $1 A$, top, netrin- 1 treatment for 30 min significantly stimulated the expression of immunoreacting, endogenous KOR proteins in mouse primary DRG neurons, which was blocked, for $\sim 80 \%$, by the translational inhibitor cyclohexamide or puromycin. The incomplete inhibition of translation by these drugs was attributable to the application of these drugs at lower concentrations to minimize their toxic effects. The specific effect on translation was also supported by the results using a transcription inhibitor actinomycin-D, which exerted no significant effect on netrin-1 stimulation of the expression of endogenous KOR. To confirm the specificity of netrin-1 to KOR, siRNA to KOR was applied in the experiment of netrin-1 stimulation (Fig. $1 \mathrm{~A}$, bottom). Clearly, silencing of the endogenous KOR gene dramatically inhibited netrin-1-stimulated KOR protein expression. The slight increase of KOR in netrin-1-stimulated, KOR-silenced cells was probably attributable to the widely recognized inability of siRNA to completely block the expression of endogenous genes. To confirm whether the effects of netrin-1 were generally true for other KOR-expressing cells, we conducted the same set of experiments in P19 cells. Similarly, KOR expression in P19 was also stimulated by netrin-1, which was also effectively inhibited by translational inhibitors and silencing of KOR but not by the transcriptional inhibitor (supplemental Fig. 1, available at www.jneurosci.org as supplemental material).

\section{Increase in functional KOR protein by netrin-1 stimulation in} primary DRG neurons

The KOR-stimulating effect of netrin-1 was found to be at the level of protein expression (Fig. 1). We then determined whether the increased KOR proteins could contribute to the biological function of KOR in neurons, such as specific ligand binding. We then examined the KOR ligand-binding patterns of primary DRG neurons using biotin-labeled dynorphin (Fig. $2 A, B$ ) and ${ }^{3} \mathrm{H}$ labeled U69,593 (Fig. 2C,D). To first establish that biotinylation on dynorphin did not affect its property as a specific KOR ligand, we examined the $K_{\mathrm{i}}$ values of dynorphin and biotin-dynorphin 
in competition experiments against ${ }^{3} \mathrm{H}$ U69,593 (Stahn et al., 1998). As shown in supplemental Figure 2 (available at www. jneurosci.org as supplemental material), both dynorphin and biotin-dynorphin exhibited a very similar $K_{\mathrm{i}}$ value (supplemental Fig. 2, available at www.jneurosci. org as supplemental material). This result justified the application of biotin-dynorphin as a specific KOR ligand in the in situ ligand binding assay. Biotin-dynorphin was then applied in subsequent experiments in which cells were treated with netrin- 1 and a panel of compounds, including inhibitors and competing ligands, as shown in Figure 2A. Clearly, dynorphin binding to the primary DRG neurons was significantly enhanced by netrin-1, which was very effectively inhibited by translation inhibitors such as cyclohexamide and puromycin but not by the transcription inhibitor actinomycin-D. With the addition of different competing KOR ligands (norBNI, U69,593 or dynorphin), binding of biotin-dynorphin to these cells was also significantly inhibited. To further validate the specific effect of netrin-1 to KOR, we applied KOR siRNA into cells, followed by ligand binding assays (Fig. $2 \mathrm{~B}$ ). Consistent with the results shown in Figure 1, KOR silencing rendered very few cells bound by dynorphin even after netrin-1 treatment. This result further supported the specific and stimulatory effect of netrin-1 on the synthesis of endogenous KOR proteins.

A quantitative ligand-binding assay using ${ }^{3} \mathrm{H}-\mathrm{U} 69,593$ (Fig. 2C) also showed that U69,593 binding to the DRG neurons was significantly enhanced. The specificity of ligand binding was verified in competition experiments in which the binding could be blocked by the antagonist nor-BNI, as well as by the cold ligand U69,593. The quantitative binding assays were also subjected to KOR silencing in the DRG neurons (Fig. 2D), which showed effective inhibition of netrin-1-stimulated KOR ligand binding by silencing the endogenous KOR.

To examine the molecular mechanisms underlying the stimulating effects of netrin-1 on KOR protein expression, the P19 cell system was used because the very limited amount of material obtained from primary DRG neurons precluded its application in a biochemical assay for quantitative measures. We first sought a direct evidence for a translational effect of netrin-1 by using a metabolic labeling procedure (Fig. $3 A$ ). To minimize the potential effects of transcription, cells were first treated with actinomycin-D before labeling with ${ }^{35}$ S-Met/Cys. The KORspecific immunoprecipitate was then monitored by determining the amounts of ${ }^{35} \mathrm{~S}$ labeling in the precipitate. It appeared that the amount of the ${ }^{35}$ S-labeled, newly synthesized KOR was significantly greater in netrin-1-treated cells beginning at the first time point, $45 \mathrm{~min}$. The effect was even more significant at $90 \mathrm{~min}$ and then gradually leveled, although the rate of newly synthesized $\mathrm{KOR}$ appeared to remain relatively high even at $4.5 \mathrm{~h}$ after netrin-1 treatment. Importantly, this effect was dramatically in- hibited by the protein synthesis inhibitors cyclohexamide and puromycin but not by the transcription inhibitor actinomycin-D. For a negative control, the amount of newly synthesized Tau was also monitored in parallel, which appeared to remain relatively constant between the mock- and netrin-1treated groups. To provide another quantitative, kinetic evidence for the specific effects of netrin-1 on KOR translation, we generated a translational reporter that contained the intact $5^{\prime}$ - and $3^{\prime}$-UTRs of KOR mRNA and examined, in parallel with the metabolic labeling experiments, the translational efficiency of this reporter after netrin-1 treatment (Fig. $3 B$ ). With the identical treatments, the kinetics of stimulation of KOR translation, detected using the translational reporter, appeared to follow closely the kinetics detected using the metabolic labeling procedure. These results unambiguously demonstrated the stimulating, or activating, effect of netrin-1 on de novo synthesis of $\mathrm{KOR}$ proteins.

\section{Netrin-1-stimulated KOR translation is through the FAK signaling pathway}

The above findings suggested that netrin-1 was an inducer for de novo KOR synthesis. Recent studies have identified FAK as the downstream mediator of netrin-1 in axon guidance ( $\mathrm{Li}$ et al., 2004; Liu et al., 2004; Ren et al., 2004). To determine whether 
(A)

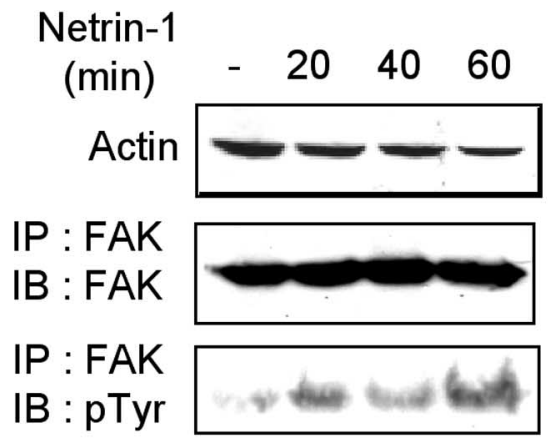

(B)
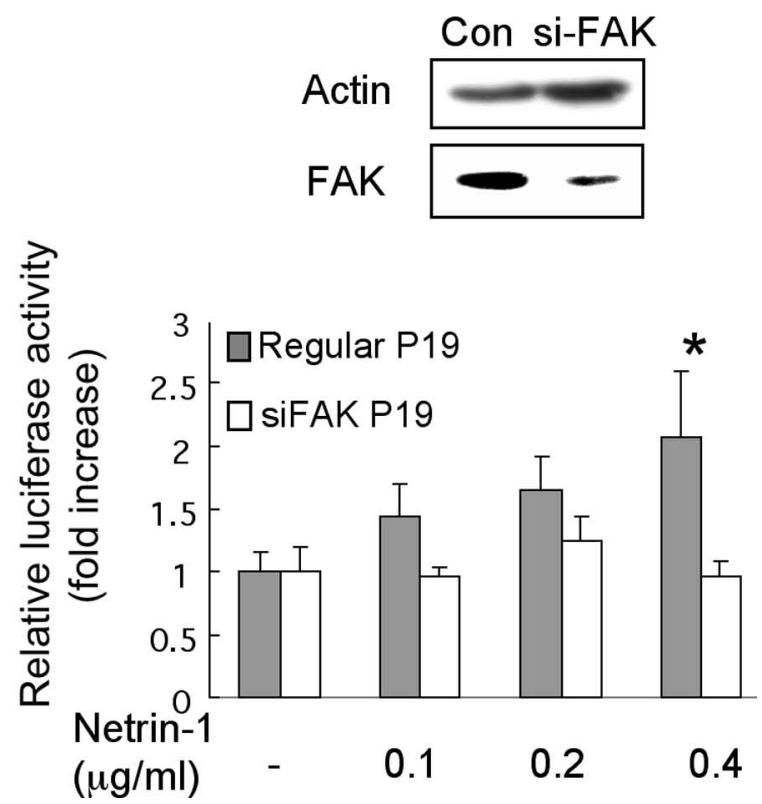

Figure 4. FAK is the downstream target of netrin- 1 in stimulating KOR translation. $\boldsymbol{A}$, Western blot analysis of netrin-1 induced phosphorylation of FAK. FAK proteins were immunoprecipitated and analyzed by anti-phosphorylated tyrosine. IP, Immunoprecipitation; IB, immunoblot. $\boldsymbol{B}$, KOR translational reporter assays in FAK-silenced P19 cells. FAK protein levels were detected on the Western blot (top). KOR reporter activities in regular or siFAK P19 cells were determined in cells treated with $0.1,0.2$, or $0.4 \mu \mathrm{g} / \mathrm{ml}$ netrin- 1 for $30 \mathrm{~min}\left({ }^{*} p<0.05\right)$. Con, control.

FAK was involved in netrin-1-stimulated translation of KOR, we monitored the behavior of the endogenous FAK and tested the biological function of FAK, specifically in regulating the expression of KOR as shown in Figure 4. It appeared that tyrosine phosphorylation of the endogenous FAK in P19 cells indeed was increased immediately (20 min) after netrin-1 treatment (Fig. 4A), suggesting that FAK was activated after netrin- 1 treatment in this experimental system. To determine whether FAK was directly involved in netrin-1-stimulated KOR expression, siRNAmediated silencing of FAK in P19 cells was performed. We generated stable P19 cell lines harvesting a vector that could constantly produce siRNA to silence the endogenous FAK in P19 cells. In addition, we applied the KOR translational reporter in the stable cell lines containing siFAK to determine, specifically, the effects of FAK on KOR translation. As shown in Figure $4 B$, this translational reporter was effectively stimulated by netrin-1, in a dose-dependent manner, in normal P19 cells. Significantly, the same reporter was no longer responsive to netrin-1 signals in
(A)

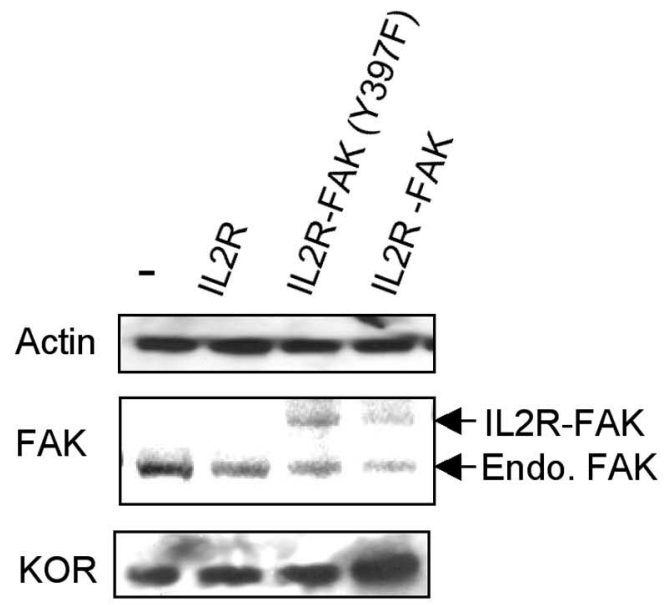

(B)

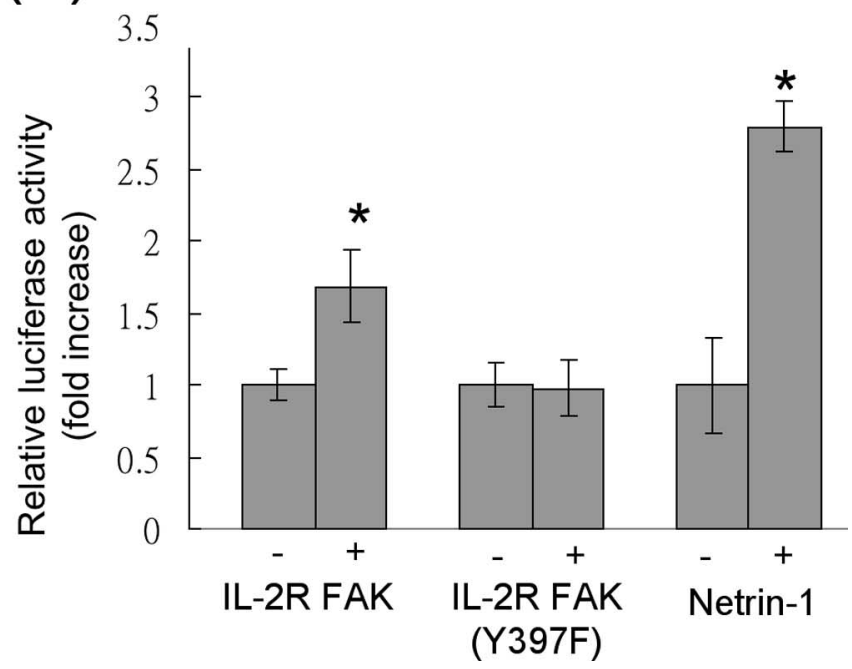

Figure 5. Constitutively active FAK upregulates KOR translation. $A$, Western blot analyses of P19 cells transfected with various forms of FAK, including a control vector IL2R, a constitutively negative FAK, IL2R-FAK-Y397F, and a constitutively active FAK, IL2R-FAK. B, KOR translational reporter activities in P19 cells transfected with the active FAK, IL2R-FAK, or the negative FAK, IL2R-FAK-Y397F, and cells treated with netrin- 1 for a comparison ( $\left.{ }^{*} p<0.05\right)$.

the FAK-silenced P19 cells. This result confirmed FAK as a key mediator of netrin- 1 in stimulating KOR translation.

To confirm the direct effect of FAK, we exploited the reported constitutively active (IL2R-FAK) and inactive (IL2R-FAKY397F) forms of FAK to bypass the input of netrin-1. P19 cells were transfected with the constitutively active or inactive FAKs, and the expression of the endogenous KOR protein was monitored as shown in Figure 5A. The results confirmed that only the constitutively active FAK (IL2R-FAK) could increase the endogenous KOR protein level. To provide additional functional evidence at the level of translation, the KOR translational reporter was used to monitor the effects of constitutively active FAK or constitutively inactive FAK, for a comparison with netrin-1 stimulation as shown in Figure $5 B$. As predicted, only the active FAK was able to increase the KOR translational reporter activity, which occurred as effectively as that triggered by netrin-1 stimulation. Altogether, these data validated that the level of endogenous KOR protein indeed was significantly and specifically elevated by netrin-1, which activated FAK, and that this occurred at the level of KOR translation. 
Netrin-1-FAK pathway regulates polysomal partition of KOR mRNA Several mechanisms were considered for the effects of netrin-1-FAK on KOR translational control. We then examined the association of KOR mRNA with polysome fractions and their translation efficiency. P19 cells were treated with netrin- 1 for 30 min. Cell extracts were then fractionated into polysomal and post-polysomal fractions from which mRNAs were isolated. The mRNA pools were then subjected to semiquantitative RT-PCR of KOR and actin as shown in Figure $6 \mathrm{~A}$. In the control cells, KOR mRNA was present in both polysomal and post-polysomal fractions. Interestingly, after netrin-1 stimulation, KOR mRNA was absent in the postpolysomal fraction and its level in the polysomal fraction was increased, suggesting that netrin-1 triggered more abundant KOR mRNAs to partition into the polysomal fraction or to be transferred from the post-polysomal to the polysomal fraction. It is also clear that the total KOR mRNA level in the total pool remained relatively constant before and after netrin-1 treatment. For a control, we monitored the distribution of $\alpha$-actin mRNA in these fractions, which was not significantly altered after netrin-1 treatment.

To examine whether KOR mRNAs loaded with polysomes were ready for translation, we determined the interaction of KOR mRNA with one component for the translational rate-limiting step, eIF4E. Anti-eIF4E was used to precipitate polysomes, which was then used to determine the amount of associated KOR mRNA (Fig. $6 B$ ). As shown in this figure, KOR mRNA association with eIF4E was increased after netrin-1 treatments. For a negative control, the associated $\alpha$-actin mRNAs was also monitored, which remained unchanged after netrin-1 treatment. It is known that mRNA translational control usually involved its UTRs. To locate the UTRs of KOR mRNA involved in netrin-1 stimulation, we generated three translational reporters of KOR $5^{\prime}$-UTR and $3^{\prime}$-UTR and determined their association with eIF4E (Fig. 6C, left). It appeared that the reporters carrying either the intact KOR 5'-3'-UTRs or the $5^{\prime}$-UTR alone were responsive to netrin-1 stimulation for association with eIF4E. Very differently, the reporter carrying the KOR $3^{\prime}$-UTR failed to respond to netrin-1 stimulation. The activities of these reporters were also determined as shown in the right panel of Figure $6 \mathrm{C}$. Consistent with the association with eIF4E, the translational reporters carrying either the intact KOR 5'-3'-UTRs or the $5^{\prime}$-UTR alone could be stimulated by netrin-1 treatment.

These data provide unambiguous evidence for netrin-1stimulated de novo KOR synthesis in primary DRG neurons and in P19 cells. The signal of netrin-1 is mediated by its downstream target FAK. Under the unstimulated condition, a significant portion of KOR mRNA is distributed in the translationally silent, post-polysomal fraction. During stimulation, the vast majority of KOR mRNA then partitions to the polysomal fraction for de novo synthesis of KOR proteins. Functionally, the newly synthesized KOR proteins in primary DRG neurons are able to bind specific ligands.
(B)
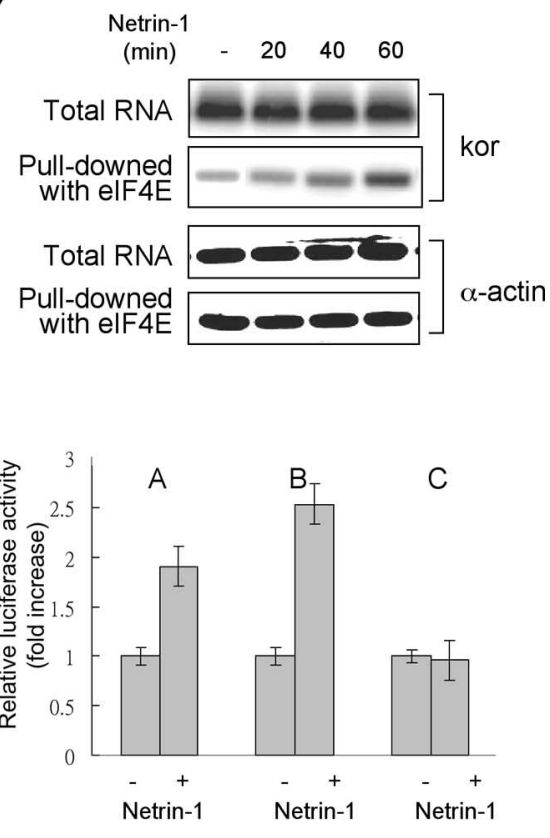

Figure 6. Netrin- 1 stimulates KOR mRNA association with polysomes. $A$, Abundance of KOR and $\alpha$-actin mRNAs in polysomal (P) versus post-polysomal (S) fractions, as well as the total mRNA pool (T). Specific associated mRNA was detected using semi-

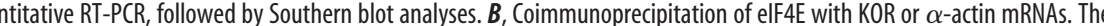
ed by anti-elF4E antibody was analyzed by semiquantitative RT-PCR. C, Semiquantitative RT-PCR analyses of elF4E-associated KOR translational reporters carrying various KOR UTRs (left). The reporter activities of the same three translational reporters were determined in parallel in P19 cells (right). luc, Luciferase.

\section{Discussion}

This study demonstrates the first example of posttranscriptional regulation of functional KOR protein synthesis by an axon guidance cue, netrin-1. The signal of netrin-1 is passed to its downstream target FAK, which triggers differential subcellular partition of KOR mRNA. Without stimulation, a significant portion of KOR mRNA stays in the post-polysomal fraction that is translationally silent. After stimulation by netrin-1, or FAK activation, the majority of KOR mRNA is then translocated to the polysomal fraction for rapid activation of translation.

Netrin-1 is known as an axon guidance cue (Kennedy et al., 1994), as well as a growth factor (Kennedy, 2000). Previous studies revealed its effects in rapid protein synthesis through the p42/ p44 MAPK signaling pathway (Campbell and Holt, 2001, 2003). Our current study identifies a novel netrin-1-triggered pathway of translational control that involves differential mRNA partition. This signaling pathway also is mediated through the FAK system. Several obvious issues have to be addressed. For instance, it is unclear how FAK acts on KOR mRNA pools. Apparently, the $5^{\prime}$-UTR of KOR is sufficient for its translational regulation by netrin-1 or by FAK, but it is unclear how the $5^{\prime}$-UTR of KOR responds to netrin-1 or FAK signals. Presumably, in unstimulated cells, a portion of KOR mRNA partitions in the inactive, post-polysomal fraction by the action of some sorts of machinery or protein complexes. FAK activation then triggers translocation of this silent KOR mRNA pool to the polysomal fraction, probably mediated by some sort of complexes stimulated by the activated FAK. It will be important to identify the target of FAK and the mediator or transporter for moving KOR mRNA around. Another interesting and important question is the physiological implication of the de novo synthesis of KOR proteins in neurons, especially in response to netrin-1 stimulation. Based on ligand 
binding assays, these newly synthesized proteins are functional. There has been no report on any potential role for KOR in axon outgrowth. Our observation, as well as the demonstrated mechanism, presents an extremely interesting scenario for future studies in terms of the physiological function of KOR in the behavior of KOR-expressing neurons.

Regulation of KOR gene expression occurs primarily during developmental stages and in cell differentiation processes ( Hu et al., 2002; Bi et al., 2003). This involves principally transcriptional events such as acquiring specific transcription factors and engaging in chromatin remodeling events (Park et al., 2005). Posttranscriptional regulation has been demonstrated for KOR, such as regulating its mRNA stability, transport, and translation (Wei and Loh, 2002; Bi et al., 2003; Wei, 2004). Although these previous studies have illustrated that the mRNA sequences could play a role in these posttranscriptional events, it was unclear how these RNA sequences were regulated and whether they responded to any environmental factors or neuronal activities. To this end, the current study has shed light on the type of signals that could regulate the expression of KOR from its mRNA. We observed that KOR mRNA could be differentially transported in differentiated P19 neurons (Bi et al., 2003) and exhibited different levels of stability (Wei et al., 2000). It is conceivable that other signal inputs might participate in this extensive network of posttranscriptional control for the production of KOR proteins. To this end, it is also of significance to identify other signal inputs for regulating the synthesis of KOR proteins, especially in neurons.

De novo protein synthesis can be particularly important for neurons. It is known that neurons engender the most extensively compartmentalized intracellular environment. It is also true that neurons require the highest degree of plasticity in terms of the amount and the type of proteins that they should produce at a given time. Although classical transport mechanisms have addressed the issue of moving various proteins or protein complexes in neurons, these mechanisms may become ineffective should neurons require some specialized proteins at a specific time. The capacity to regulate translation of specific proteins in neurons could potentially equip the cells with a greater plasticity in terms of producing specific types of proteins that are needed only when cells are stimulated. The fact that a significant portion of KOR mRNA is dormant in the control cells and that it can be rapidly activated for protein synthesis by environmental cues would suggest an intriguing role for KOR in neuronal behaviors other than receiving opioid drugs.

\section{References}

Bi J, Hu X, Loh HH, Wei LN (2001) Regulation of mouse $\kappa$ opioid receptor gene expression by retinoids. J Neurosci 21:1590-1599.

Bi J, Hu X, Loh HH, Wei LN (2003) Mouse kappa-opioid receptor mRNA differential transport in neurons. Mol Pharmacol 64:594-599.

Campbell DS, Holt CE (2001) Chemotropic responses of retinal growth cones mediated by rapid local protein synthesis and degradation. Neuron 32:1013-1026.

Campbell DS, Holt CE (2003) Apoptotic pathway and MAPKs differentially regulate chemotropic responses of retinal growth cones. Neuron 37:939-952.

Gupta P, Huq MD, Khan SA, Tsai NP, Wei LN (2005) Regulation of corepressive activity of and HDAC recruitment to RIP140 by site-specific phosphorylation Mol Cell Proteomics 4:1776-1784.

Hu X, Bi J, Loh HH, Wei LN (2002) Regulation of mouse kappa opioid receptor gene expression by different $3^{\prime}$-untranslated regions and the effect of retinoic acid. Mol Pharmacol 62:881-887.

Hu X, Chen Y, Farooqui M, Thomas MC, Chiang CM, Wei LN (2004) Suppressive effect of receptor-interacting protein 140 on coregulator binding to retinoic acid receptor complexes, histone-modifying enzyme activity, and gene activation. J Biol Chem 279:319-325.

Kennedy TE (2000) Cellular mechanisms of netrin function: long-range and short-range actions. Biochem Cell Biol 78:569-575.

Kennedy TE, Serafini T, de la Torre JR, Tessier-Lavigne M (1994) Netrins are diffusible chemotropic factors for commissural axons in the embryonic spinal cord. Cell 78:425-435.

Kim CS, Hwang CK, Choi HS, Song KY, Law PY, Wei LN, Loh HH (2004) Neuron-restrictive silencer factor (NRSF) functions as a repressor in neuronal cells to regulate the mu opioid receptor gene. J Biol Chem 279:46464-46473.

Kraus J, Borner C, Giannini E, Hickfang K, Braun H, Mayer P, Hoehe MR, Ambrosch A, Konig W, Hollt V (2001) Regulation of mu-opioid receptor gene transcription by interleukin-4 and influence of an allelic variation within a STAT6 transcription factor binding site. J Biol Chem 276:43901-43908.

Law PY, Wong YH, Loh HH (1999) Mutational analysis of the structure and function of opioid receptors. Biopolymers 51:440-455.

Li W, Lee J, Vikis HG, Lee SH, Liu G, Aurandt J, Shen TL, Fearon ER, Guan JL, Han M, Rao Y, Hong K, Guan KL (2004) Activation of FAK and Src are receptor-proximal events required for netrin signaling. Nat Neurosci 7:1213-1221.

Liu G, Beggs H, Jurgensen C, Park HT, Tang H, Gorski J, Jones KR, Reichardt LF, Wu J, Rao Y (2004) Netrin requires focal adhesion kinase and Src family kinases for axon outgrowth and attraction. Nat Neurosci 7:1222-1232.

Minami M, Satoh M (1995) Molecular biology of the opioid receptors: structures, functions and distributions. Neurosci Res 23:121-145.

Mukhopadhyay D, Houchen CW, Kennedy S, Dieckgraefe BK, Anant S (2003) Coupled mRNA stabilization and translational silencing of cyclooxygenase-2 by a novel RNA binding protein, CUGBP2. Mol Cell 11:113-126.

Park SW, Li J, Loh HH, Wei LN (2002) A novel signaling pathway of nitric oxide on transcriptional regulation of mouse $\kappa$ opioid receptor gene. J Neurosci 22:7941-7947.

Park SW, Huq MD, Loh HH, Wei LN (2005) Retinoic acid-induced chromatin remodeling of mouse $\kappa$ opioid receptor gene. J Neurosci 25:3350-3357.

Pasternak GW (2004) Multiple opiate receptors: deja vu all over again. Neuropharmacology 47 [Suppl 1]:312-323.

Piper M, Salih S, Weinl C, Holt CE, Harris WA (2005) Endocytosisdependent desensitization and protein synthesis-dependent resensitization in retinal growth cone adaptation. Nat Neurosci 8:179-186.

Ren XR, Ming GL, Xie Y, Hong Y, Sun DM, Zhao ZQ, Feng Z, Wang Q, Shim S, Chen ZF, Song HJ, Mei L, Xiong WC (2004) Focal adhesion kinase in netrin-1 signaling. Nat Neurosci 7:1204-1212.

Stahn R, Schafer H, Kernchen F, Schreiber J (1998) Multivalent sialyl Lewis $\mathrm{x}$ ligands of definite structures as inhibitors of E-selectin mediated cell adhesion. Glycobiology 8:311-319.

Stucky CL, Shin JB, Lewin GR (2002) Neurotrophin-4: a survival factor for adult sensory neurons. Curr Biol 12:1401-1404.

Tsai NP, Wu YC, Chen JW, Wu CF, Tzeng CM, Syu WJ (2006) Multiple functions of 10036 in the regulation of the pathogenicity island of enterohaemorrhagic Escherichia coli O157:H7. Biochem J 393:591-599.

Wei LN (2004) Retinoids and receptor interacting protein 140 (RIP140) in gene regulation. Curr Med Chem 11:1527-1532.

Wei LN, Loh HH (2002) Regulation of opioid receptor expression. Curr Opin Pharmacol 2:69-75.

Wei LN, Hu X, Bi J, Loh H (2000) Post-transcriptional regulation of mouse kappa-opioid receptor expression. Mol Pharmacol 57:401-408.

Zheng JQ, Kelly TK, Chang B, Ryazantsev S, Rajasekaran AK, Martin KC, Twiss JL (2001) A functional role for intra-axonal protein synthesis during axonal regeneration from adult sensory neurons. J Neurosci 21:92919303. 